INTERNATIONAL JOURNAL FOR NUMERICAL METHODS IN ENGINEERING

Int. J. Numer. Meth. Engng 2011; 01:1-20 Prepared using nmeauth.cls [Version: 2002/09/18 v2.02]

\title{
Numerical Simulation of Flapping Wings using a Panel Method and a High-Order Navier-Stokes Solver
}

\author{
P.-O. Persson ${ }^{1, *}$, D. J. Willis ${ }^{2}$, and J. Peraire ${ }^{3}$ \\ ${ }^{1}$ Dept. of Mathematics, University of California, Berkeley, CA, USA \\ 2 Dept. of Mechanical Engineering, University of Massachusetts, Lowell, MA, USA \\ 3 Dept. of Aeronautics and Astronautics, Massachusetts Institute of Technology, Cambridge, MA, USA
}

\begin{abstract}
SUMMARY
The design of efficient flapping wings for human engineered micro aerial vehicles (MAVs) has long been an elusive goal, in part due to the large size of the design space. One strategy for overcoming this difficulty is to use a multi-fidelity simulation strategy appropriately balances computation time and accuracy. We compare two models with different geometric and physical fidelity. The low-fidelity model is an inviscid doublet lattice method with infinitely thin lifting surfaces. The high-fidelity model is a high-order accurate discontinuous Galerkin Navier-Stokes solver which uses an accurate representation of the flapping wing geometry. To compare the performance of the two methods, we consider a model flapping wing with an elliptical planform and an analytically prescribed spanwise wing twist, at size scales relevant to MAVs. Our results show that in many cases, including those with mild separation, low fidelity simulations can accurately predict integrated forces, provide insight into the flow structure, indicate regions of likely separation and shed light on design relevant quantities. But for problems with significant levels of separation, higher fidelity methods are required to capture the details of the flow-field. Inevitably high-fidelity simulations are needed to establish the limits of validity of the lower fidelity simulations. Copyright (C) 2011 John Wiley \& Sons, Ltd.
\end{abstract}

KEY WORDS: Flapping flight, panel method, discontinuous Galerkin, Navier-Stokes, Arbitrary Lagrangian-Eulerian.

* Correspondence to: P.-O. Persson, Dept. of Mathematics, University of California, Berkeley, Berkeley, CA 94720-3840, U.S.A. (persson@berkeley.edu)

Contract/grant sponsor: U.S. Air Force Office of Scientific Research (AFOSR), Multi-University Research Initiative: Biologically-Inspired Flight for Micro Air Vehicles; contract/grant number: 001425594000

Contract/grant sponsor: U.S. Air Force Office of Scientific Research (AFOSR), Young Investigator Award; contract/grant number: FA9550-10-1-0229

Contract/grant sponsor: Director, Office of Science, Computational and Technology Research, U.S. Department of Energy; contract/grant number: DE-AC02-05CH11231

Copyright (C) 2011 John Wiley \& Sons, Ltd. 


\section{INTRODUCTION}

Flapping propulsion strategies that are observed in nature are a result of many years of refinement and evolution. While nature presents a diversity of flapping winged animals, it is difficult to assess the relative importance of flapping parameters (flapping frequency, amplitude, upstroke retraction, forward-aft flapping etc.), shape characteristics (wing camber, twist, spanwise bending, etc) and skeletal structure (wing stiffness, material properties, skeletal layout) by simply observing and recording the wingbeat kinematics of these animals. For example, most birds exploit a wing retraction during the upstroke, however, it is not clear from experimental observations of natural flyers what the importance of those kinematics are, and whether a human designed micro aerial vehicle (MAV) would benefit from mimicking this behavior or whether a related, but different set of kinematics would suffice. Furthermore, there is a strong dependence between the various possible flapping parameters. For example, modulating flapping frequency independently of wing shape adjustments provides a partial and potentially misleading view of the performance envelope. It is unfeasible to develop a full appreciation of the flapping flight design space from nature alone. Thus, the use of analytical and computational models which are capable of capturing the essential mechanisms of flapping flight becomes a necessity. The use of these models allows us to characterize the role of different flapping parameters, including those commonly observed in natural flapping flight.

Computational modeling and simulation have been used to understand the kinematics and aerodynamics of flapping winged animals and vehicles $[1,2,3,4,5,6]$. At one end of the modeling spectrum are efficient, low-fidelity methods such as linear potential flow methods (momentum disk and vortex methods [7, 8, 9], lifting line [1, 10, 11], vortex/doublet lattice methods [12, 13], and panel methods [14, 2, 3, 15]). These lower fidelity simulations are computationally efficient and require limited user involvement in the description and representation of the geometry. The solution time for these methods is typically on the order of seconds or minutes on a desktop computer. Unfortunately, many of these lowerfidelity simulations are considered unreliable for modeling flapping wings due to the physical assumptions associated with potential flow. Specifically, these methods employ limited or no viscous modeling, and as a result, critical effects such as flow separation are ignored, or at best poorly represented. At the other end of the spectrum are the higher fidelity Navier-Stokes methods which incorporate a complete physical model of the problem $[4,5,3]$. These methods, despite offering an opportunity to model the flow accurately, are prohibitively expensive for examining every possible combination of flapping kinematics. While these methods are capable of accounting for the detailed flow characteristics, the solution comes at a significant cost, both in setting up the geometry and in simulating the flow.

Several research efforts have proposed and developed modeling strategies that exploit variable- or multiple-fidelity physics and geometry representations $[16,3,17]$. We have proposed a multi-fidelity computational framework $[6,18,16]$ that uses a collection of potential flow methods (Wake-Only Energetics, Lifting Line and Panel Methods) with differing levels of geometric representation, and a high-order Navier-Stokes method. We focus here on two independent methods from our multi-fidelity framework for modeling flapping flight; a panel method and a high-order accurate Navier-Stokes method. By examining and understanding how the introduction of viscosity at the higher fidelity level impacts the flow-field and performance of a flapping wing, we can more confidently apply our multi-fidelity framework to design more effective flapping wings.

Int. J. Numer. Meth. Engng 2011; 01:1-20 
In this paper, we compare initial results of a lower fidelity model (potential flow panel method) with higher fidelity simulations (Navier-Stokes). Here, we consider an analytically described three-dimensional flapping wing and present a small collection of three-dimensional computations. The flapping motions we consider has varying levels of prescribed active twist modulation $[14,19,20,21,22]$. A primary objective of this paper is to understand the applicability of each fidelity level in different regimes of the design space. Although the Reynolds number in the Navier-Stokes simulations $(R e=3000)$ is in characteristic of insect flight, we expect this study to provide general insight into the applicability of the numerical methods across a wide range of animals, including insects, bats and birds. The lower Reynolds number regime in the Navier-Stokes simulations is dominated by viscous laminar flow, which will, in general be more susceptible to flow separation and strong viscous effects. This highly viscous regime is expected to produce a range of flow behavior that can not be captured by the inviscid methods. We find for flapping kinematics where there is limited to no flow separation, our potential flow model predicts integrated quantities such as vertical and horizontal forces with good trend accuracy. Even when there is mild separation, the potential flow accurately predicts trends, most likely because the Kutta condition in the potential flow method still accurately accounts for the wing-bound circulation. There is however, a noticeable degradation in the predictive capability of our potential flow model for cases where the flow separates from the wing and does not re-attach. While it is easy to observe the inconsistencies between our potential flow predictions and the Navier-Stokes simulations aposteriori, it is a challenge to predict when potential flow results will adequately represent reality and which will not. We find that one such apriori indicator is the presence of a strong leading edge suction peak and subsequent significant adverse pressure gradient. A strong adverse pressure gradient after the leading edge suction peak was a strong indication of flow separation.

\section{PROBLEM STATEMENT}

Our study is based on a prescribed motion flapping wing, with parameters than allow us to vary the lift and thrust forces to obtain a wide variety of flow regimes.

\subsection{Wing Geometry and Flapping Motion}

The wing geometry is a symmetric HT13 airfoil with an elliptical planform. The baseline wing kinematics are derived analytically to obtain approximate feathering conditions. This motion is then modified by introducing a twist scaling factor that we use to get thrust-producing flapping kinematics. In addition, we vary the angle of attack in order to get a range of lift forces.

The wing geometry is described in terms of a fixed reference frame $X, Y, Z$. We start by considering a wing with elliptical planform in the $X Y$-plane:

$$
Y= \pm \frac{1}{2} \sqrt{1-\left(\frac{X}{b}\right)^{2}} .
$$

The maximum normalized chord at the wing centerline is $c=1$ and the wing tip-to-tip span is $2 b=10$. An HT13 airfoil is selected for the entire wing span, resulting in a maximum wing thickness of $t=0.065$ at the wing centerline. 

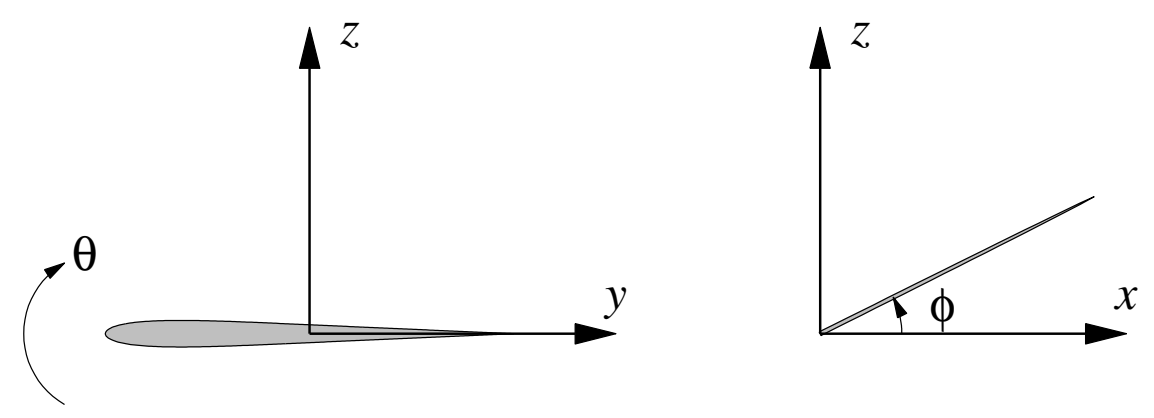

Figure 1. The wing geometry is an elliptic extrusion of an HT13 wing section. The flapping motion is described in terms of a flapping angle $\phi$ and a twist angle $\theta$.

The flapping motions occur symmetrically about a hinge located at the wing centerline (see figure. 1).

We prescribe the symmetric wing motion using a flapping angle at the wing centerline hinge given by

$$
\phi(t)=A_{\phi} \cos \omega t
$$

where $t$ is the time, $A_{\phi}$ is the flapping amplitude and $\omega$ the flapping angular frequency. In addition, a wing twist angle is prescribed as a function of the span location. At the distance $X$ from the centerline of the wing, the twist angle is

$$
\theta(t, X)=\varepsilon(a(X) \sin \omega t+b(X) \cos \omega t)
$$

where the twist scaling factor $\varepsilon \in[0,1]$ is a parameter that controls the amount of spanwise twist, and the coefficients $a(X), b(X)$ are chosen to locally align the wing with the flow when $\varepsilon=1$, as described below.

Consider a point $P=(X, Y, 0)$ located on the leading edge of the wing, with approximate velocity

$$
v_{P}=-X \dot{\phi}+Y \dot{\theta}
$$

The feathered condition for a free-stream flow of $u_{\mathrm{inf}}$ in the positive $Y$-direction is then

$$
v=u_{\text {inf }} \tan \theta \approx u_{\text {inf }} \theta .
$$

Combining the above expressions with equation (1) for the wing profile, we get

$$
\frac{1}{2} \sqrt{1-\left(\frac{X}{b}\right)^{2}} \dot{\theta}-u_{\text {inf }} \theta=X \dot{\phi}=-X A_{\phi} \omega \sin \omega t
$$

or

$$
A(X) \dot{\theta}-\theta=B(X) \sin \omega t, \quad \text { where } \quad A(X)=-\frac{\sqrt{b^{2}-X^{2}}}{4 u_{\infty} b}, \quad B(X)=\frac{X \phi_{0} \omega}{u_{\infty}}
$$




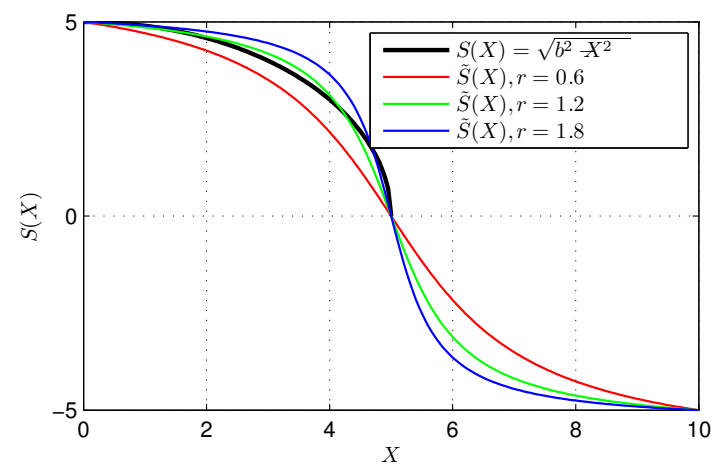

Figure 2. Comparison of the function $S(X)=\sqrt{b^{2}-X^{2}}$, which is non-smooth at $X=b$ and not defined for $X>b$, with the approximate functions $\tilde{S}(X)$ from (9) using different values of $r$ and $b=5$.

Solving this equation, we can determine the coefficients $a(X)$ and $b(X)$ in expression (3) after setting $\varepsilon=1$,

$$
a(X)=\frac{B(X)}{A(X)^{2} \omega^{2}+1}, \quad b(X)=\frac{B(X) A(X) \omega}{A(X)^{2} \omega^{2}+1} .
$$

Because of the elliptical planform, the function $A(X)$ is not differentiable at the tip of the wing $(X=b)$. In order to obtain this tip singularity, we modify the flapping motion locally and replace the numerator in $A(X)$ by a smooth approximation

$$
\sqrt{b^{2}-X^{2}} \approx \tilde{S}(X) \equiv \frac{\arctan (r(b-X))}{\arctan (r b)} b
$$

where we empirically choose the appropriate value $r=1.2$, see Fig. 2 for an illustration. Therefore, $A(X)$ becomes

$$
A(X)=-\frac{\tilde{S}(X)}{4 u_{\infty} b}
$$

Note that this expression is well defined and smooth beyond the wing $(X>b)$ and will be used below to construct a smooth embedding of the deforming domain.

The resulting flapping motion is illustrated in figure 3. We note that this is motion is parametrized in terms of the twist scaling factor $\varepsilon$ and the global angle of attack. Although this is not an optimized flapping motion, it is adequate for the purposes of comparing the simulation results obtained with our two different computational models.

\subsection{Flow Properties and Test Matrix}

The free stream flow has a Mach number of 0.1 , and for the Navier-Stokes simulations, the wing root chord Reynolds number is 3,000. We have used nine sets of parameters for our simulations, corresponding to three freestream angles of attack $\alpha=0,5$,and 10 degrees, and three twist scaling factors $\varepsilon=1.0,0.75$, and 0.5 . The twist scaling factors were chosen to provide reasonable levels of local wing incidence alleviation during flapping. $\varepsilon=0.5$ represents 


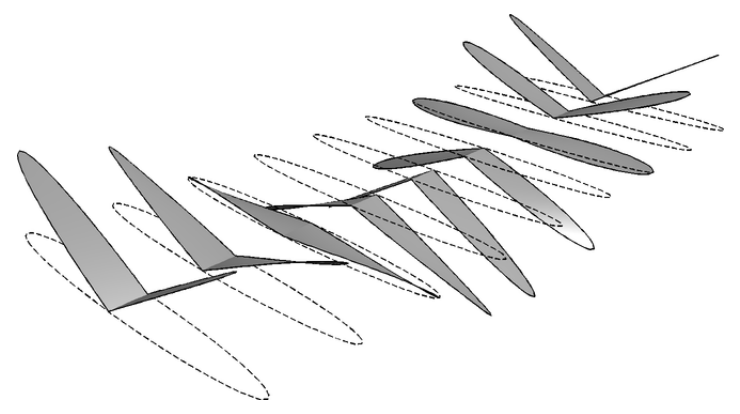

Figure 3. The prescribed flapping motion for the elliptic wing (feathered condition $\varepsilon=1.0$ ).

a case with high local angles of attack during much of the flapping cycle. While more aggressive cases than $\varepsilon=0.5$ could be considered (eg: $\varepsilon=0.0$ ), the resulting motions have poor lift and thrust production efficiency due to overly aggressive local angles of attack. .

The wing geometry, frequency, and amplitude are held constant for all simulations, with a flapping amplitude of $A_{\phi}=30^{\circ}$ and an angular frequency of $\omega=2 \pi / 20$. This corresponds to a reduced frequency $k=c \omega /\left(2 u_{\text {inf }}\right)=\pi / 20 \approx 0.16$. This reduced frequency is lower than most low Reynolds number natural flyers.

\section{COMPUTATIONAL MODELS}

We solve our flapping wing problem using two techniques - the potential flow panel method code FastAero, and the Navier-Stokes based discontinuous Galerkin code 3DG. Here, we give an overview of the two methods and a description of the discretization and solution techniques we use. For more details, we refer to previous work by us and other authors.

\subsection{Panel Method: FastAero}

FastAero $[23,6,18,16]$ is a boundary element or aerodynamic panel method $[24,2,25]$ for solving unsteady, linear potential flow. Despite the simplifications introduced by linear potential flow (inviscid, irrotational, incompressible flow), panel methods are powerful and accurate when analyzing the aerodynamic behavior of streamlined shapes such as wings, fuselages and faired bodies [2]. Although, it is reasonable to expect flapping wings to experience some flow separation, we assume that large regions of separated flow are not desirable in cruise flapping flight due to the increased energetic demands. As such, for the most desirable cruise configurations, we expect our panel method to be an adequate model.

The three-dimensional panel method, FastAero, [23] is an unsteady, accelerated, panel method with vortex particle wakes. FastAero includes options for Dirichlet boundary conditions (modeling potential flow around both thick wings and [2]) and Neumann doublet lattice method formulations (modeling potential flow around thin wings [12]). Linear strength basis functions [26] are used in FastAero to represent the distribution of source and/or doublet strength on each of the triangular elements. The integration of the linear source and doublet influences, 
at a given point in the domain, is performed using analytical panel integration [26]. The boundary conditions on each panel are satisfied using a Galerkin weighted residual method, with the integrals being numerically approximated by multi-point Gaussian Quadrature [27]. While computationally more costly than traditional constant collocation panel methods, our approach has several benefits. First, the high-order representation of the source and doublet distributions leads to a more robust and accurate solution. Second, high-order panel methods are more tolerant for irregular discretizations, which are likely to occur in unsteady flapping wing geometries. Third, the tangential components of the velocity at the body, and thus the surface pressure, is computed by evaluating the gradient of the surface potential on each panel rather than using finite difference methods. This simpler surface gradient computation enables the use of triangular panels and also results in efficient post-processing routines that do not impose geometric constraints on the model (eg: ordered, quadrilateral panels).

Linear potential flow approaches often vary in their representation of the trailing wake the thin sheet of vorticity shed behind each lifting surface. In FastAero we model and convect the shed vorticity using a vortex particle method [28, 29, 30,31]. We use the vortex particle method because of its relative simplicity and automatic handling of the wake evolution in complex flows.

One of the enabling features FastAero for analyzing flapping flight is the low computational cost. The computational cost of a single FastAero time-step scales approximately linearly with the number of unknowns in the problem $(\mathcal{O}(n \log (n)))$ due to the use of iterative matrix solution methods combined with approximate matrix vector product calculations. A precorrected-FFT method (pFFT [32]) is used to accelerate the solution of the linear system using the GMRES [33] iterative method, while a fast mutlipole tree algorithm $[34,35]$ is used to reduce the complexity of evaluating the influences of the vortex particle wake on the body, the wake and the surroundings. Not only does this acceleration help to efficiently solve the boundary integral equation, it also allows us to use a larger number of discrete surface elements as required to obtain grid converged solutions.

Due to the inviscid assumption in potential flow analyses, a Kutta condition must be imposed at the sharp trailing edge of the wing to prescribe the strength of the shed vorticity at each time-step [36]. In our implementation, we can select between a linear Kutta condition, based on the continuity of the potential jump strength, or a non-linear Kutta condition, which imposes pressure equality at the trailing edge. Here, we use the iterative, non-linear, pressure Kutta condition. Although this pressure Kutta condition is more expensive computationally, it is able to more rigorously satisfy the actual condition at the trailing edge of the flapping wing.

Once the potential flow equation is solved for a given timestep, the surface velocity, pressure and forces can be easily obtained by post-processing the potential solution. The total surface velocity is computed by adding the tangential components of the surface potential gradient and the tangential component of the free stream velocity. Once the surface velocity is determined, the unsteady Bernoulli equation is used to determine the surface pressure.

\subsection{Discontinuous Galerkin Arbitrary Lagrangian-Eulerian (ALE) Navier-Stokes: 3DG}

For the high-fidelity simulations, we use the 3DG code [37, 38, 39, 40, 41, 42], which implements a high-order accurate discontinuous Galerkin method to solve the compressible Navier-Stokes equations on unstructured meshes of tetrahedra. Although the flow is nearly incompressible, the use of a compressible flow formulation has the advantage of providing high-order accuracy

Int. J. Numer. Meth. Engng 2011; 01:1-20 
in time with regular ODE time integrators. Furthermore, we have found that the ILU preconditioner employed scales well on parallel computers even though the connections across subdomains are neglected. This behavior can be explained by the fact that small times are required for accuracy which in turn produce better conditioned global matrices.

High-order methods are advantageous for applications requiring low numerical dispersion and high time accuracy. The DG method produces stable discretizations of the convective operator for any order discretization, thus avoiding the need for additional stabilization or filtering. For the flows considered, with a fairly low Reynolds number of 3,000, high-order methods are capable of fully resolving all the scales present in the flow and hence accurately predict flow separation and reattachment.

The fully unstructured simplex meshes allow for arbitrary geometries and domains, and for efficient adapted meshes that focus the computational resources on relevant regions. To handle the moving boundaries and the deforming domains, we use a mapping-based Arbitrary Lagrangian-Eulerian (ALE) formulation [43].

Our solvers have been verified in several ways before. In [44], it was shown that transitional flows in non-deforming domains modeled using Implicit Large Eddy Simulations agree well with experiments and other simulations. Fully turbulent flows with RANS modeling were considered in [42], and the ALE formulation for deforming domains was validated for a number of twodimensional cases in [43]. While it is hard to find experimental data for the complex flapping motions and flow regimes considered in this work, we show that our results converge in both space and time when the orders of the schemes are increased. This is a good indication that the simulations accurately predict these laminar flows.

3.2.1. Spatial Discretization using Curved Mesh Elements In order to obtain maximum geometric flexibility, we employ fully unstructured meshes of triangles and tetrahedra. We use the symmetry of the problem to only simulate one half of the domain. The outer boundary of the domain is a half-cylinder, with a radius of 10 chord lengths and a height of 20 chord lengths. We generate all the surface meshes (the wing, the symmetry plane, and the cylindrical outer surfaces) in parametrized form using the DistMesh triangular mesh generator [45]. The tetrahedral volume mesh is then generated by a Delaunay refinement based code [46]. The resulting mesh has about 43,000 nodes and 231,000 tetrahedral elements for the half-domain. A mesh size function is used to focus the resolution on the area around the wings, in particular at the leading and the trailing edges, as well as in the wake. The mesh used in the computations is shown in figure. 4.

To obtain high-order accuracy in the simulations, the straight-sided tetrahedral meshes must be modified to account for the curved domain boundaries. This mesh modification is accomplished using the nonlinear elasticity approach proposed in Ref. [47], whereby the layer of elements around the wing is considered to be a deformable elastic solid obeying a nonlinear neo-Hookean constitutive law. This approach leads to a well-shaped mesh in which the facets of the elements in contact with the surface are curved to align with the true geometry.

3.2.2. ALE Formulation We solve the compressible Navier-Stokes equations in conservative form, which can be written as a system of conservation laws

$$
\frac{\partial u}{\partial t}+\nabla \cdot F^{\mathrm{i}}(u)-\nabla \cdot F^{\mathrm{v}}(u, \nabla u)=0
$$



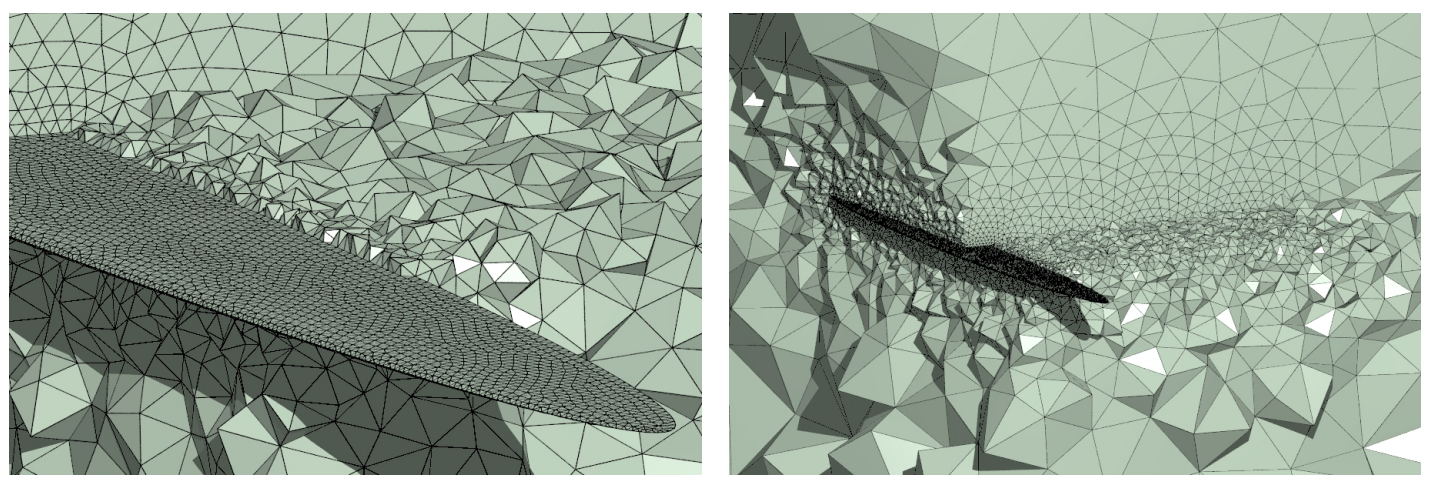

Figure 4. A tetrahedral mesh for the domain around the elliptic wing pair.

where $u=\left(\rho, \rho u_{1}, \rho u_{2}, \rho u_{3}, \rho E\right)^{T}$ is the solution vector of conserved components, with density $\rho$, velocities $u_{1}, u_{2}, u_{3}$, and total energy $E$, and $F^{\mathrm{i}}$ and $F^{\mathrm{v}}$ are the inviscid and viscous flux functions, respectively.

To account for the deforming domain, we employ the mapping based Arbitrary LagrangianEulerian (ALE) formulation proposed in Ref. [43]. It uses a mapping $x=x(X, t)$ between the coordinates $X$ in a reference domain and the corresponding coordinates $x$ in the deforming physical domain. We introduce the mapping deformation gradient $G$ and the mapping velocity $\nu$ as

$$
G=\frac{\partial \boldsymbol{x}}{\partial \boldsymbol{X}}, \quad \boldsymbol{\nu}=\frac{\partial \boldsymbol{x}}{\partial t} .
$$

In addition, we denote the Jacobian of the mapping by $g=\operatorname{det}(G)$. This allows us to transform the original system (11) into an equivalent system of conservation laws in the reference domain:

$$
\left.\frac{\partial u_{X}}{\partial t}\right|_{X}+\nabla_{X} \cdot F_{X}^{\mathrm{i}}\left(u_{X}\right)-\nabla \cdot F_{X}^{\mathrm{v}}\left(u_{X}, \nabla_{X} u_{X}\right)=0
$$

where the time derivative is at a constant $X$ and the spatial derivatives are with respect to the $X$ variables. The transformed vector of conserved quantities and corresponding fluxes in the reference space are

$$
\begin{aligned}
& u_{X}=g u \\
& F_{X}^{\mathrm{i}}=g G^{-1} F^{\mathrm{i}}-u_{x} G^{-1} \boldsymbol{\nu} \\
& F_{X}^{\mathrm{v}}=g G^{-1} F^{\mathrm{v}}
\end{aligned}
$$

and

$$
\nabla u=\nabla_{X}\left(g^{-1} u_{X}\right) G^{-1}=\left(g^{-1} \nabla_{X} u_{X}+u_{X} \nabla_{X}\left(g^{-1}\right)\right) G^{-1}
$$

This formulation has the advantage that it solves the transformed equations on fixed computational mesh and the complexity of the deforming domain is accounted for in the transformation mapping. This approach leads to arbitrary high orders of accuracy in both space and time. We refer to [43] for additional details. 


\subsection{Mapping function}

The reference configuration for the wing flapping motion, is the neutral horizontal and untwisted wing position. A time dependent smooth mapping function $\boldsymbol{x}=\boldsymbol{x}(\boldsymbol{X})$ is created such that, at the wing surface, it matches the wing prescribed motion. Ideally, we would like the mapping that is well-behaved away from the wing such that is volume preserving $(g=1)$. A volume preserving mapping not only simplifies the ALE equations, but we have found that it also makes the scheme much less sensitive to the instabilities described in [43] and allow us to solve without the additional considerations required for satisfying the geometric conservation law.

While there are many ways to find such a mapping, we use an approach based on shearing, blending, and scaling, as described below.

1. The deformation of a point $(X, Y, Z)$ due to the rotations $\phi(t)$ and $\theta(t, X)$ is handled by a vertical offset in the $Z$-direction by $X \sin \phi+Y \sin \theta$, and a compression in the $X, Y$-plane of $X \cos \phi, Y \cos \theta$, respectively.

2. To reduce the amount of deformation away from the wing, we use smooth blendings for the $X, Y$ coordinates such that the grid deformation changes from that prescribed by the wing close to the wing surface to a constant value for large $|X|,|Y|$.

3 . Finally, the $Z$-coordinate is scaled to make the deformation volume preserving $(g=1)$.

More precisely, we first define a smoothed ramp function by

$$
\mathcal{R}(x)= \begin{cases}0 & \text { if } x<0 \\ 0.5 & \text { if } x>1 \\ x^{6}-3 x^{5}+\frac{5}{2} x^{4} & \text { otherwise }\end{cases}
$$

which we use to define limited $X, Y$ coordinates by

$$
\begin{gathered}
X_{\lim }=X-b \cdot(2 \mathcal{R}(X / b-1)+\mathcal{R}(|X| / b-1)), \\
Y_{\lim }=Y-b \cdot(2 \mathcal{R}(Y / b-1)+\mathcal{R}(|Y| / b-1)) .
\end{gathered}
$$

Note that $X_{\lim }=X$ when $|X|<b$ and $X_{\lim }=b / 2$ when $|X|>2 b$, and similarly for $Y_{\lim }$.

We use these limited coordinate functions to reduce the effect of the shearing, but also to make the coefficient $B(X)$ in (7) better behaved away from the wing surface. The remaining coefficients are defined as before in (10) and (8):

$$
\begin{aligned}
A(X) & =-\frac{\tilde{S}(X)}{4 u_{\infty} b}, & B(X) & =\frac{X_{\lim } \phi_{0} \omega}{u_{\infty}}, \\
a(X) & =\frac{B(X)}{A(X)^{2} \omega^{2}+1}, & b(X) & =\frac{B(X) A(X) \omega}{A(X)^{2} \omega^{2}+1} .
\end{aligned}
$$

and the final mapping has the form:

$$
\boldsymbol{x}(\boldsymbol{X}, t)=\left[\begin{array}{c}
X-(1-\cos \phi) X_{\lim } \\
Y-(1-\cos \theta) Y_{\lim } \\
Z /\left(\left(1-(1-\cos \phi) X_{\lim }^{\prime}\right) \cdot\left(1-(1-\cos \theta) Y_{\lim }^{\prime}\right)\right)+X_{\lim } \sin \phi+Y_{\lim } \sin \theta
\end{array}\right]
$$

where the scaling of the $Z$ coordinate is such that $g=\operatorname{det}(G)=1$. Note that the deformation gradient $G=\frac{\partial \boldsymbol{x}}{\partial \boldsymbol{X}}$ is lower triangular, and we use analytical differentiation to find all its matrix entries as well as the grid velocity $\partial \boldsymbol{x} / \partial t$. 
3.3.1. High-Order DG Spatial Discretization The 3DG flow solver is based on the highorder Discontinuous Galerkin (DG) method with tetrahedral mesh elements and nodal basis functions. Here, we use nodal basis functions and cubic polynomials (degree $p=3$ ). We also consider $p=1$ and $p=2$ for one of the test cases, in order to assess the discretization accuracy and demonstrate grid convergence. The governing equations are written as a system of first order conservation laws

$$
\begin{aligned}
\frac{\partial u}{\partial t}+\nabla \cdot F^{\mathrm{i}}(u)-\nabla \cdot F^{\mathrm{v}}(u, q) & =0 \\
q & =\nabla u,
\end{aligned}
$$

which are discretized using a discontinuous Galerkin method [48]. The spatial domain $\Omega$ is discretized into a triangulation $\mathcal{T}_{h}$, and we seek solutions in the finite element spaces

$$
\begin{aligned}
V_{h} & =\left\{v \in\left[L^{2}(\Omega)\right]^{5}|v|_{K} \in\left[\mathcal{P}_{p}(K)\right]^{5}, \quad \forall K \in \mathcal{T}_{h}\right\}, \\
\Sigma_{h} & =\left\{r \in\left[L^{2}(\Omega)\right]^{5 \times 3}|r|_{K} \in\left[\mathcal{P}_{p}(K)\right]^{5 \times 3}, \quad \forall K \in \mathcal{T}_{h}\right\},
\end{aligned}
$$

where $\mathcal{P}_{p}(K)$ is the space of polynomial functions of degree at most $p \geq 0$. The DG formulation then becomes: find $u_{h} \in V_{h}$ and $q_{h} \in \Sigma_{h}$ such that for all $K \in \mathcal{T}_{h}$, we have

$$
\begin{aligned}
& \int_{K} \frac{\partial u_{h}}{\partial t} v d x-\int_{K}\left(F^{\mathrm{i}}\left(u_{h}\right)-F^{\mathrm{v}}\left(u_{h}, q_{h}\right)\right) \cdot \nabla v d x+\int_{\partial K}\left(\hat{F}^{\mathrm{i}}-\hat{F}^{\mathrm{v}}\right) v d s=0, \quad \forall v \in\left[\mathcal{P}_{p}(K)\right]^{5}, \\
& \int_{K} q_{h} \cdot r d x=-\int_{K} u_{h} \nabla \cdot r d x+\int_{\partial K} \hat{u} r \cdot n d s, \quad \forall r \in\left[\mathcal{P}_{p}(K)\right]^{5 \times 3} .
\end{aligned}
$$

Here, the inviscid numerical fluxes $\hat{F}^{\mathrm{i}}$ are approximated using the method of Roe[49]. For the viscous fluxes $\hat{F}^{\mathrm{v}}$, we use the compact discontinuous Galerkin (CDG) scheme[37] and choose $\hat{u}$ and $\hat{F}^{\mathrm{v}}$ according to

$$
\begin{aligned}
\left(\hat{F}^{\mathrm{v}}\right)^{e} & =\left\{\left\{F^{\mathrm{v}}\left(u_{h}, q_{h}^{e}\right) \cdot \boldsymbol{n}\right\}\right\}+C_{11} \llbracket u_{h} \boldsymbol{n} \rrbracket+\boldsymbol{C}_{12} \llbracket F^{\mathrm{v}}\left(u_{h}, q_{h}^{e}\right) \cdot \boldsymbol{n} \rrbracket \\
\hat{u} & =\left\{\left\{u_{h}\right\}\right\}-\boldsymbol{C}_{12} \cdot \llbracket u_{h} \boldsymbol{n} \rrbracket .
\end{aligned}
$$

Here, $\{\{\}\}$ and $\llbracket \rrbracket$ denote the average and jump operators across the interface [37]. We set $C_{11}=0$ at all internal faces, and $C_{11}=10 / h$ at the Dirichlet boundaries where $h$ is a typical dimension of the element in the direction normal to the boundary, and $\boldsymbol{C}_{12}=\boldsymbol{n}^{*}$. Here, $\boldsymbol{n}^{*}$ is the unit normal to the interface, which can be taken to have arbitrary sign provided the sign is the same for the to elements sharing that face. The "edge" fluxes $q_{h}^{e}$ are computed by solving the equation

$$
\int_{K} q_{h}^{e} \cdot r d x=-\int_{K} u_{h} \nabla \cdot r d x+\int_{\partial K} \hat{u}^{e} r \cdot n d s, \quad \forall r \in\left[\mathcal{P}_{p}(K)\right]^{5 \times 3},
$$

where

$$
\hat{u}_{h}^{e}= \begin{cases}\hat{u}_{h} & \text { on edge } e, \text { given by equation }(31) \\ u_{h} & \text { otherwise }\end{cases}
$$


The boundary conditions are imposed in terms of the fluxes $\hat{u}$ and $\hat{F}^{\mathrm{i}}, \hat{F}^{\mathrm{v}}$. For more details on the scheme we refer to Ref. [37].

The discrete finite element spaces $V_{h}, \Sigma_{h}$ are represented by nodal Lagrange basis functions within each tetrahedral element. All volume and face integrals are computed by specialized Gaussian integration rules for simplex elements, up to an order of precision $3 p$. We note that due to the choice of $\hat{u}$ in the CDG scheme (31), the variables $q_{h}$ can be evaluated explicitly within each element and eliminated from the global equation system. This results in a discretized scheme that only depends on $u_{h}$. If $U$ denotes the global solution vector containing the nodal values of $u_{h}$ in each elements, we can write a nonlinear system of ODEs:

$$
M \frac{d U}{d t}=R(U)
$$

with mass matrix $M$ and residual vector $R(U)$.

3.3.2. DIRK Temporal Discretization We integrate the system of ODEs (34) in time using a third-order accurate three-stage Diagonally Implicit Runge-Kutta (DIRK) method [50], where the solution is advanced from time $t_{n}$ to $t_{n+1}$ using the scheme:

$$
\begin{aligned}
& M K_{i}=R\left(t_{n}+c_{i} \Delta t, U_{n}+\Delta t \sum_{j=1}^{s} a_{i j} K_{j}\right), \quad i=1, \ldots, s \\
& U_{n+1}=U_{n}+\Delta t \sum_{j=1}^{s} b_{j} K_{j}
\end{aligned}
$$

where $s=3$ and the Runge-Kutta tableaux is given by:

\begin{tabular}{r|c|cccc}
$\alpha$ & $\alpha$ & 0 & 0 & $\alpha=0.435866521508459$ \\
$\tau_{2}$ & $\tau_{2}-\alpha$ & $\alpha$ & 0 & $\tau_{2}=(1+\alpha) / 2$ \\
1 & $b_{1}$ & $b_{2}$ & $\alpha$ & $b_{1}=-\left(6 \alpha^{2}-16 \alpha+1\right) / 4$ \\
$c$ & $A$ \\
\hline & $b^{T}$
\end{tabular}$=\begin{aligned} b_{1} & b_{2} & \alpha & b_{2}=\left(6 \alpha^{2}-20 \alpha+5\right) / 4\end{aligned}$

This one-step scheme is also L-stable, which we have found to be a requirement for the simulations presented in this paper. Since our ODE scheme is implicit, it requires inversion of matrices of the form $M-\alpha \Delta t d R / d U$. This is accomplished by using a preconditioned NewtonKrylov technique, as described below. All of our simulations are done using this three-stage scheme, but for the temporal convergence study we also consider schemes with $s=1$ and $s=2$ stages, see [50] for details.

3.3.3. Parallel Newton-Krylov Solvers The systems of equations produced by the DG discretization are typically very large. We use polynomials of degree $p=3$, which gives 20 degrees of freedom per tetrahedron and solution component. Since we have 5 solution components, this gives a total number of degrees of freedom around 23 million. In addition, the Jacobian matrices tend to be less sparse than those typically obtained with low-order methods. Although we use an efficient compressed compact storage format [39], each Jacobian 
has about 7 billion entries, and requires $55 \mathrm{~GB}$ of storage. It is clear that parallel computers are needed, both for storing these matrices and to perform the computations.

The parallel 3DG code [40] is based on the MPI interface, and runs on parallel computer clusters. The domain is decomposed using the METIS software [51] and the discretization and matrix assembly is done in parallel. In order to solve the linear systems in the Newton method, we use an ILU-preconditioned CGS solver. To maximize the performance of the preconditioner, we order the elements using the Minimum Discarded Fill (MDF) algorithm [39].

Most of the simulations were done on 16 compute nodes with 8 cores each, or a total of 128 processors. The simulation times in this setting are on the order of 24 hours per 100 timesteps, although this depends on the problem, the timestep, and the tolerances in the Newton and the Krylov solvers.

\section{RESULTS}

We first present a general characterization of the flowfield as predicted by the Navier-Stokes and the panel method models. Then, we present the results for the time-evolution of the integrated vertical and horizontal forces. Finally, we present the results for the wake structure.

\subsection{Characterizing the Flow}

We use the both the panel method and the Navier-Stokes computations to model the flow and predict the unsteady pressure differential between the top and bottom surface of the wing (figure. 6). From the pressure differential results, we observe a distinct difference between the approximately feathered wing $(\epsilon=1)$ and the flapping kinematics for which a more aggressive local incidence is prescribed $(\epsilon=0.75$ and $\epsilon=0.5)$. For the feathered case, the pressure differential distribution over the surface of the wing is almost unchanged over the duration of the wingbeat cycle. Even when the global incidence angle is increased, the wing still remains similarly loaded throughout the wingbeat. The pressure differential predicted by both the panel method and the Navier-Stokes simulation are similar across the wingspan in these less aggressive flight regimes. By contrast, we observe that the pressure differential varies more when the wing twist is prescribed in a more aggressive manner $(\epsilon=0.75$ and $\epsilon=0.5)$. In these simulations, the pressure differential varies cyclicly with the the flapping motions, producing a more negative pressure differential during the downstroke and a more positive pressure differential during the upstroke. This overall trend is consistent in both computational predictions; however, in the Navier-Stokes results we observe a pronounced effect of flow separation on the wing surface pressure differential distribution. In particular, the Navier-Stokes simulation differs from the panel method prediction during the downstroke of the most aggressive wing twist angles, $\epsilon=0.5$, where significant flow separation is observed. These pressure differential results also indicate that the wing global incidence, $\alpha$, impacts the pressure distribution across the entire wing; however, there is a higher pressure differential effect in the outboard regions of the wing.

For most of the computational cases considered, the panel method predicts a large suction peak along the leading edge of the wing. This pressure peak leads to a higher adverse pressure gradient at locations directly downstream of the leading edge, indicating the potential for flow separation. This peak is a consequence of the potential flow model enforcing an attached flow 
condition around the sharp leading edge of the wing. As seen in the Navier-Stokes simulations (figures. 7-8), the less aggressive cases (low $\alpha$ and $\epsilon=1.0,0.75$ ) exhibit consistently attached flow, while the more aggressive cases (high $\alpha$ and $\epsilon=0.75,0.5$ ) result in leading edge flow separation due to the inability of viscous flows to conform to the extreme flow curvature around the leading edge. This leading edge separation manifests in the surface pressure differential plots computed using the Navier-Stokes solver (figure. 6). From the Navier-Stokes simulations we observe that flow separation causes a mitigation of the leading edge pressure peak, and a center of pressure that moves rearward toward the trailing edge.

The results of the Navier-Stokes simulations are shown in figures. 7-8. In figure. 7, entropy iso-surfaces with Mach number color contours are generated to illustrate regions of flow separation and wake vortex structures. The Mach number throughout the domain remains below 0.25 for all cases, which indicates that compressibility effects are likely not important. For the least aggressive cases considered (wing twist parameter, $\epsilon=1,0.75$, and global incidence, $\alpha=0$ ), the wing is approximately aligned with the flow for the duration of the flapping cycle. In these two cases, the flow remains attached throughout the wingbeat cycle. When the wing twist parameter is most aggressive $(\epsilon=0.5)$, for the same global incidence angle $(\alpha=0)$, the Navier-Stokes simulation predicts flow separation in the outboard region of the wing during both the upstroke and the downstroke. As the global incidence angle increases $(\alpha=5,10)$, we observe a strong effect of global wing incidence on the flowfield. For these cases, the wing has a more aggressive angle of attack during the downstroke than the upstroke, and consequently, greater levels of flow separation are observed during the downstroke than the upstroke. The first half of the downstroke is generally characterized by attached flow, while the second half of the downstroke and the first quarter of the upstroke exhibit separated flow. For the most aggressive case $(\epsilon=0.5, \alpha=10)$, the flow is separated for most of the flapping cycle, with the exception of the middle portions of the upstroke and the initial portion of the downstroke. Overall, the Navier-Stokes results suggest that the cases chosen for this study exhibit many of the flow characteristics of interest.

\subsection{Time History of Integrated Forces: Panel Method and Navier-Stokes Simulation}

The time evolution of vertical forces for both the panel method and the Navier-Stokes simulation is shown in figure. 5 . The vertical force direction is defined perpendicular to the freestream flow ( $x$-direction), and parallel with the $z$-dimension or gravity. These force histories correlate with the flowfield results shown in figure. 6 and figure. 7. For the approximately feathered geometry $(\epsilon=1, \alpha=0)$, both the Navier-Stokes solver and FastAero predict low vertical force magnitudes. For both computational methods, we observe a multi-peakforce history, indicating that the wing is not perfectly feathered, rather there is some nonzero incidence during parts of the wingbeat cycle. While both methods predict similar force histories, there are differences between the two predictions, which can be attributed to the viscous nature of the flow. When the wing global incidence angle is increased, we observe a comparable multi-peak time-history of the vertical force in both modeling approaches, except that, for these non-zero incidence cases the average vertical force is offset by a mean value. At the highest incidence angle $(\alpha=10)$, for the same twist motion $(\epsilon=1)$, the force timehistories indicate a fundamental difference. The Navier-Stokes simulation predicts an erratic time history corresponding to flow separation, while the panel method solver continues to predict smooth force histories as in the previous cases. 

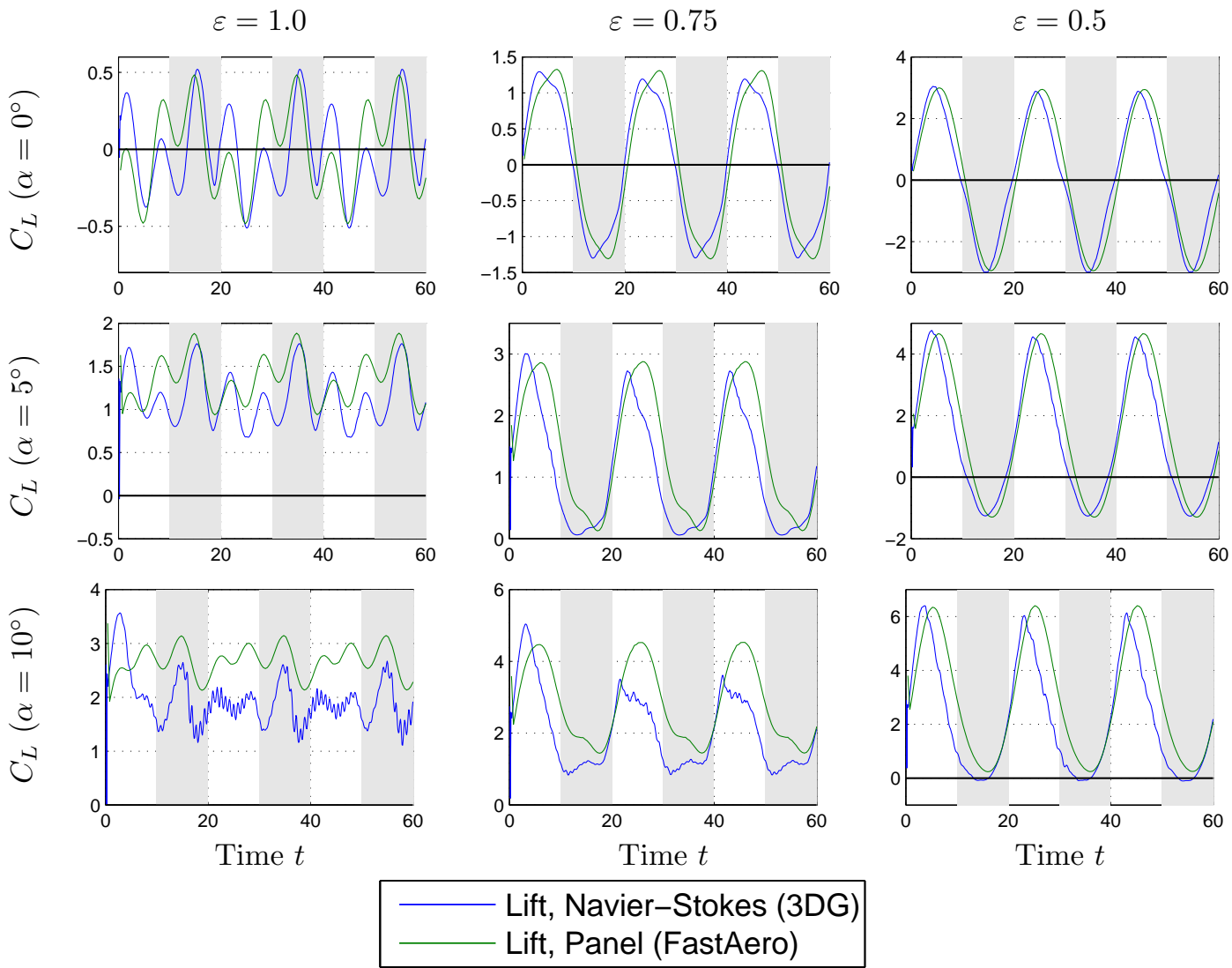

Figure 5. The lift coefficients $C_{L}$ computed by the two simulation codes for the nine cases $\alpha=0,5$, 10 , and $\varepsilon=1.0,0.75,0.5$.

When the wing twist parameter, $\epsilon$, is reduced $(\epsilon=0.75$ and $\epsilon=0.5)$ the vertical force has a different time series characteristic. In both the Navier-Stokes and the panel method results, we generally observe a single, dominant vertical force peak during the downstroke and a similar single peak during the upstroke. Additionally, we also observe that increases in the global wing incidence angle, $\alpha$, produce a similar offset as previously seen in the vertical force time-history. As the global incidence of the wing increases, the average vertical force also tends to increase. For most of the computations performed, the panel method trends well with the Navier-Stokes simulations. Once the simulations reach a periodic steady state, the panel method consistently over-predicts the vertical force maximum value. In most cases, this difference is minor, with only one case indicating a significant discrepancy between the panel method and the NavierStokes simulation results $(\epsilon=0.75, \alpha=10)$. The entropy-isosurface plots indicate that this particular case exhibited significant flow separation through much of the downstroke. Similarly, the force-histories predicted by the panel method and the Navier-Stokes solver are comparable during the first half of the downstroke; however, during the second half of the downstroke the 


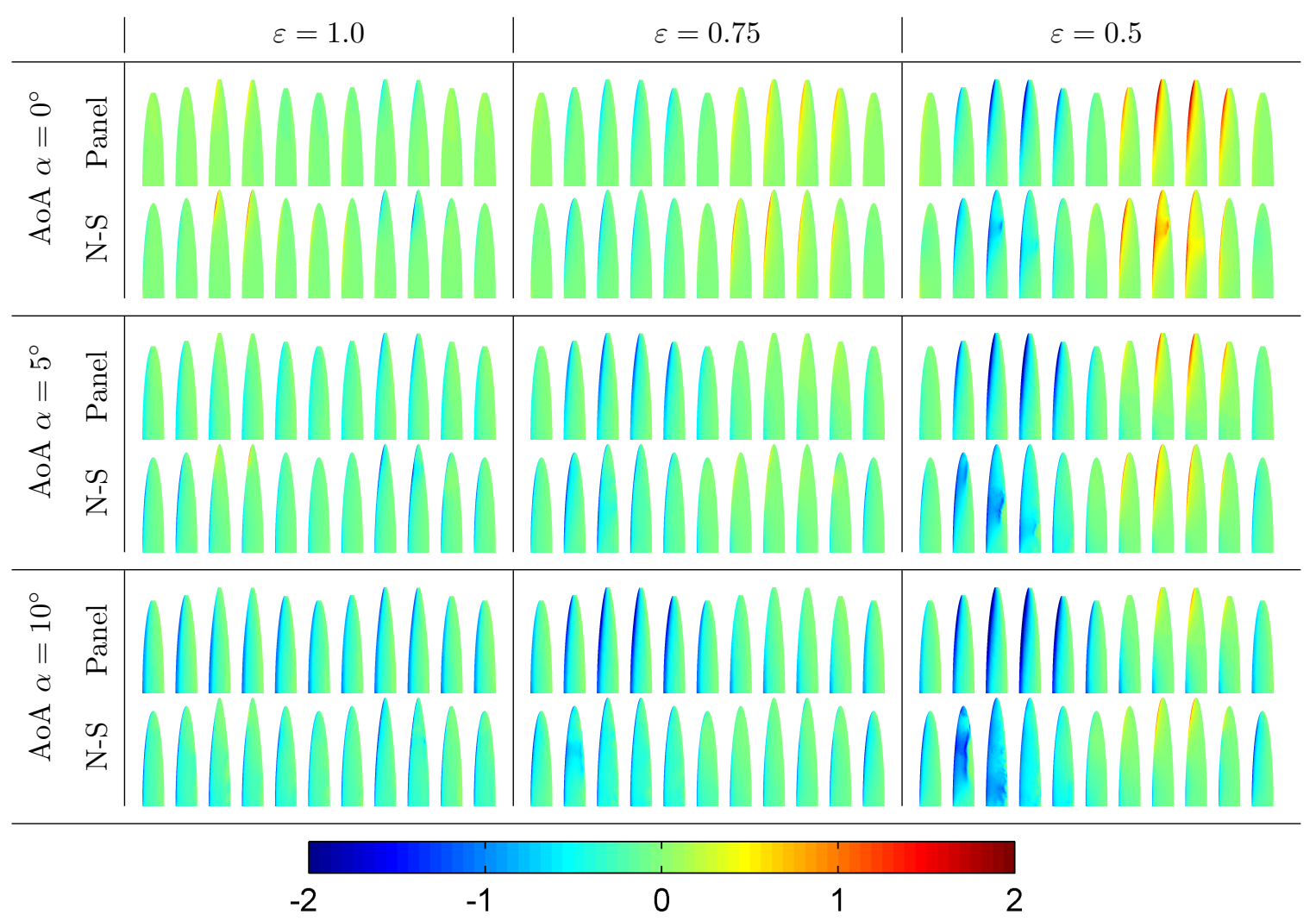

Figure 6. The panel method computed wing surface pressure differential is shown for a series of snapshots during the flapping cycle for each angle of attack $\alpha$ and twist scaling factors $\varepsilon=1.0,0.75,0.5$ from left to right. The pressure differential indicates a large pressure differential near the leading edge of the wing, in particular in the outboard locations of the wing.

forces return to the zero-load condition later than the Navier-Stokes simulations indicate. This phase shift in the force recovery is noticeable for all cases where the wing-twist parameter is not-feathered $(\epsilon \neq 1)$. Based on the entropy isosurfaces, the actual flow exhibits vortex shedding and large scale separation during the second half of the downstroke, suggesting this phase shift in the force prediction is associated with the presence of a vortex structure above the wing.

The predictions for the panel method and Navier-Stokes time evolution of the horizontal forces are shown in figure. 8. Despite the lack of viscous force predictions in the panel method, the trends and structure of the force history compares well with the Navier-Stokes method for most of the cases examined. For all of the cases, there is a noticeable mean offset between the magnitude of the Navier-Stokes and panel method horizontal force predictions, due mostly to the lack of viscous modeling in the panel method. While this offset can be estimated using integrated skin friction drag corrections, the underlying potential flow method is incapable of capturing these viscous force contributions. While the time histories of the horizontal force 


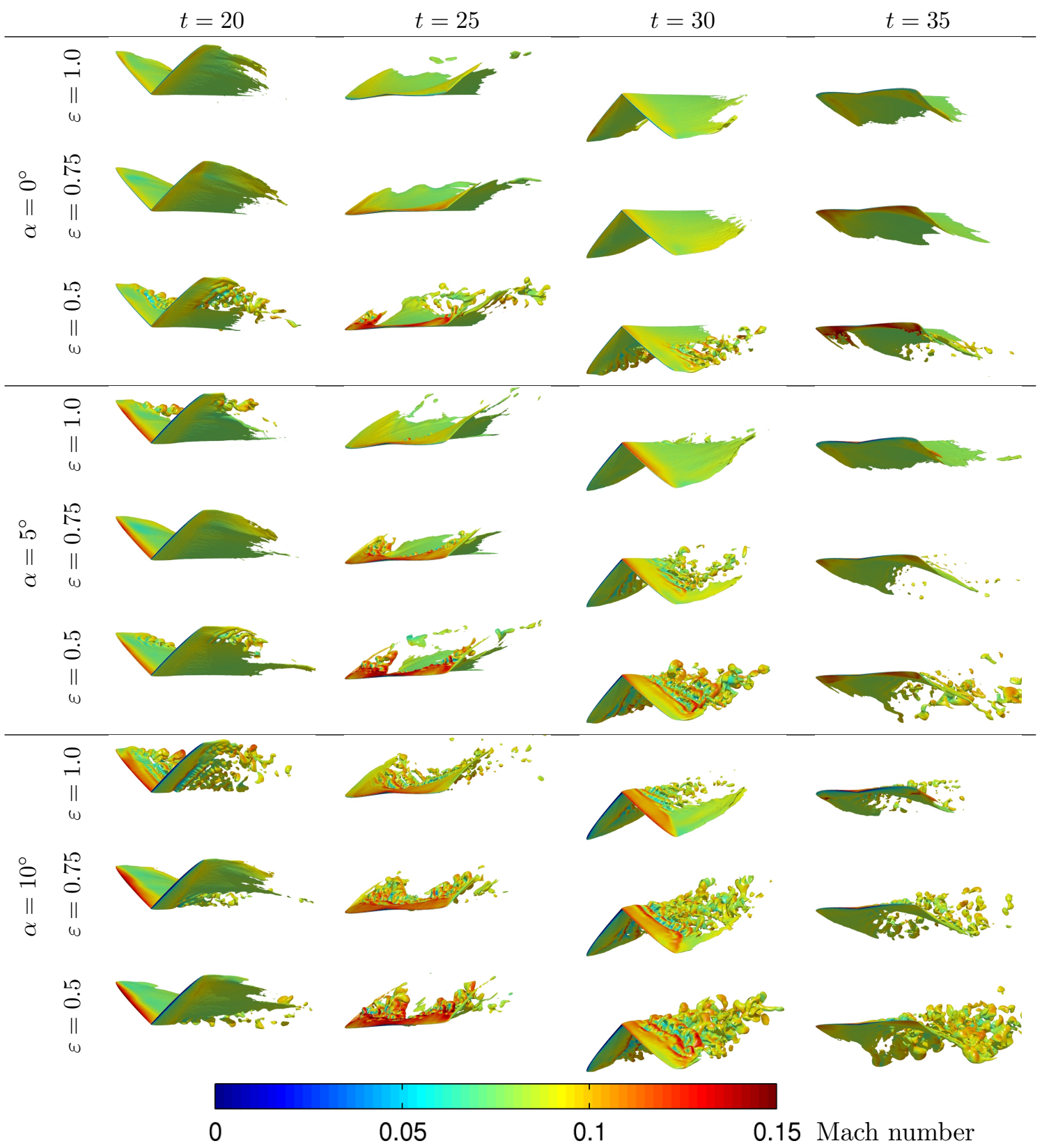

Figure 7. The flowfield around the flapping wing pair computed by the 3DG Navier-Stokes code, visualized as Mach number color plots on isosurfaces of the entropy. The plots correspond to the three cases of angle of attack (top to bottom) and the times $t=20, t=25$, and $t=30$ (left to right). 

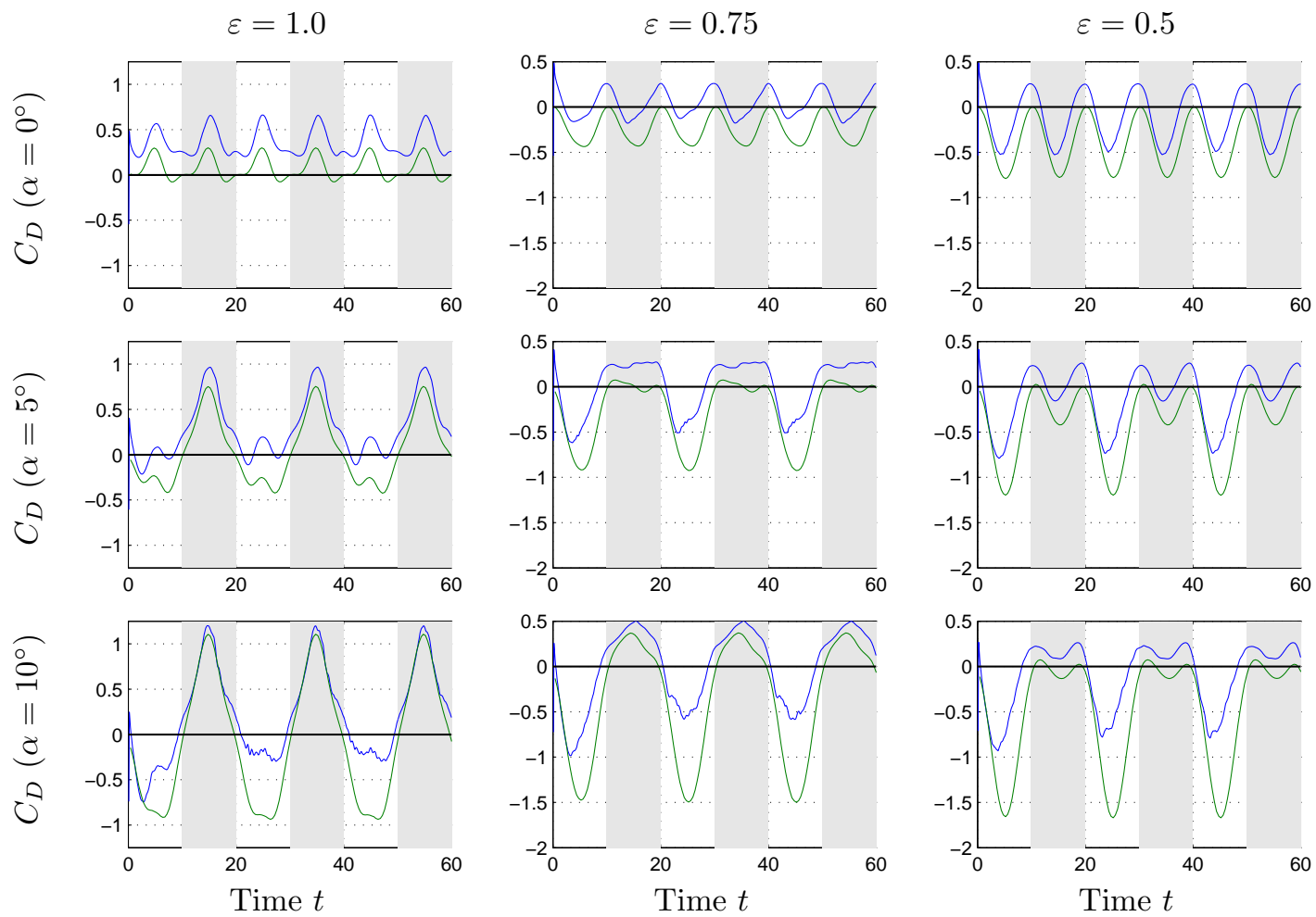

Drag, Navier-Stokes (3DG) Drag, Panel (FastAero)

Figure 8. The drag coefficients $C_{D}$ computed by the two simulation codes for the nine cases $\alpha=0,5$, 10 , and $\varepsilon=1.0,0.75,0.5$.

appear to be trend accurate, the integrated drag and therefore the propulsive efficiency will also be poorly predicted by the potential flow panel method unless an adequate viscous flow correction is made. In addition, there is a phase shift, with peak drag forces being consistently predicted later in the panel method than in the Navier-Stokes simulation. Finally, the panel method exhibits smooth time-history predictions as before; however, the NavierStokes simulation indicates portions of the flapping cycle where forces are negatively impacted by flow separation.

4.2.1. Grid convergence In order to get an indication of the accuracy of the Navier-Stokes computations, we perform a temporal and a spatial convergence study where we solve the same problem using various timesteps as well as various polynomial orders of the spatial approxiating space.

We consider the case $\alpha=5^{\circ}, \varepsilon=0.5$, and show in figure 9 the resulting forces for the two timesteps $\Delta t=0.1$ and $\Delta t=0.1 / 5=0.02$. We note that the computed forces are very close, 

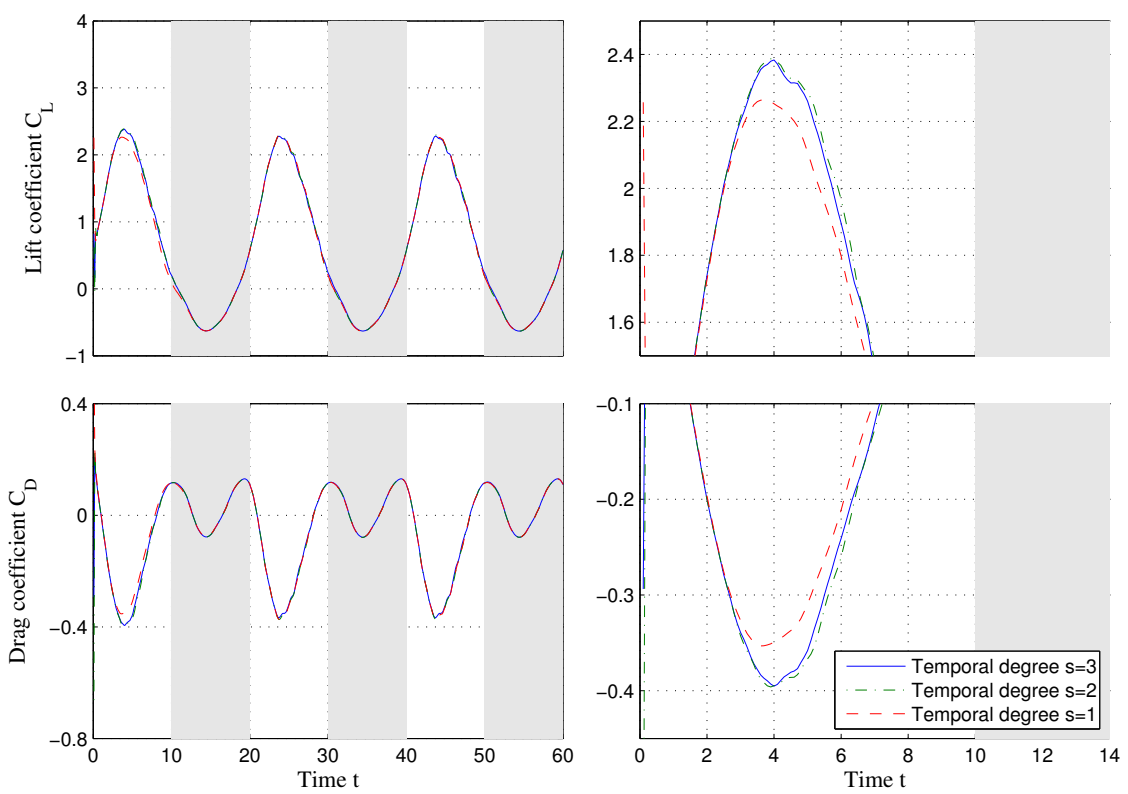

Figure 9. Temporal convergence for the Navier-Stokes simulation, for the $\alpha=5^{\circ}, \varepsilon=0.5$ case with spatial discretization using polynomial degrees $p=3$. Three different Runge-Kutta schemes were used, with number of stages $s=1,2,3$, and the small differences between $s=2$ and $s=3$ indicates that the computed forces are accurate.

with a maximum error of less than $2 \%$ relative to the largest values.

Similarly, to examine the spatial convergence, we show the forces computed with polynomial orders $p=1, p=2$, and $p=3$ in figure 10 . We note that the $p=1$ case is highly inaccurate, likely because the flow is under-resolved. However, the difference between $p=2$ and $p=3$ are relatively small, which indicates that the $p=3$ simulations are well-resolved and sufficiently accurate for our purposes.

\subsection{Wake Structure and Characteristics}

In our panel method model, vorticity is shed from the trailing edge only. While leading edge shedding is also possible in panel methods, this was not considered in the present analysis. We make the underlying assumption that efficient flapping vehicles will have a minimum of flow separation. In figure. 11, a-d we show the equivalent spatial trace of the wake circulation as an equivalent doublet sheet. This wake-surface plot illustrates the wake structure. Regions of higher circulation magnitude indicate indicate regions of higher wing loading. Regions of rapid surface-tangential change in the circulation are indicative of increased vorticity distribution magnitude. Contours of constant wake circulation provide an approximation of the wake vortex structures. For the cases with more aggressive wing incidence $(\epsilon \neq 1)$ we observe a double ladder structure - with clear tip, starting and stopping vortices during the cycle. 

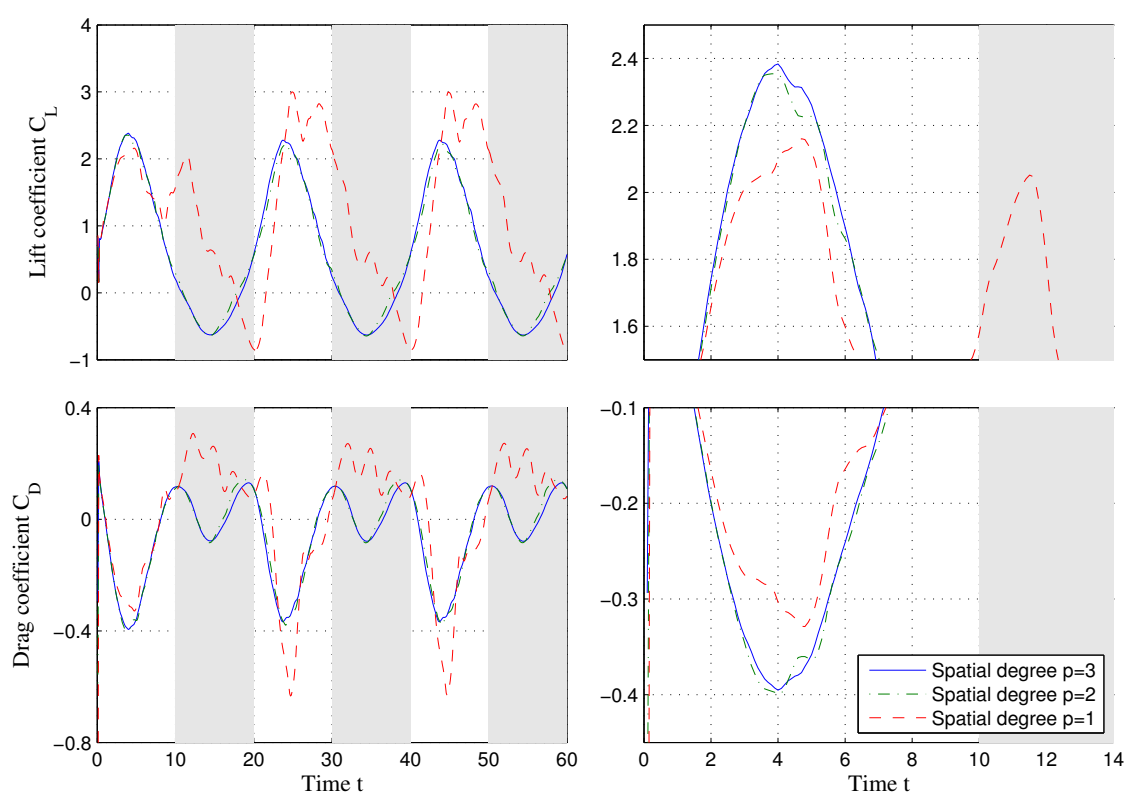

Figure 10. Spatial convergence for the Navier-Stokes simulation, for the $\alpha=5^{\circ}, \varepsilon=0.5$ case with temporal discretization using the three-stage Runge-Kutta scheme. Three different polynomial orders were used on the same grid, and the small differences between $p=2$ and $p=3$ indicates that the computed forces are accurate.

\section{DISCUSSION}

\subsection{General Discussion of Simulation Results}

The Navier-Stokes simulation shows a wide range of flow behavior for the cases tested. In the less aggressive cases the flow remains attached, while, the more aggressive cases are characterized by separated flow for a large portion of the downstroke. Because of the potential flow assumptions, the panel method does not predict or model flow separation. The effects of viscosity are also observed in the Navier-Stokes simulation during the first half of the downstroke, where a vortical structure develops and stays over the suction surface of the wing. This raises the concern that the potential flow method, despite predicting the integrated forces accurately, does not model the appropriate flow physics or accurate flow structures for these cases. Despite not modeling the separated flow physics, the panel method does indicate regions where flow separation is likely. These suspect regions are characterized by large adverse pressure gradients, similar to those observed in the leading edge region of the wing in the more aggressive flapping cases. The large spike in low pressure at the leading edge and subsequent pressure recovery for the remainder of the chordwise flow indicates regions of likely separation; however, because attached flow is assumed in the potential flow approach, separated flow is not modeled. Obviously, if the details of the flow surrounding the wing are important, then the Navier-Stokes simulation capability is essential; however, if basic insights into the flow are 


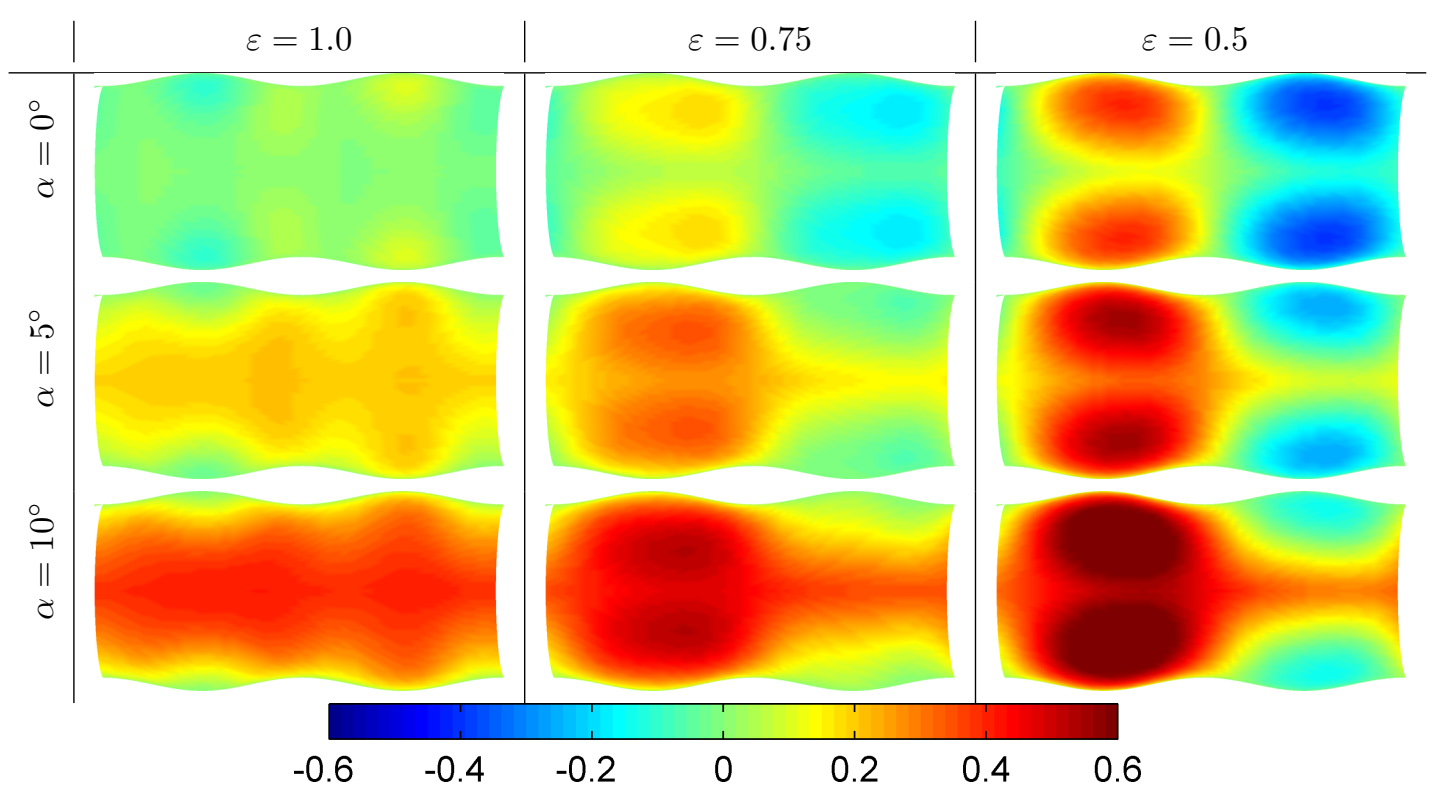

Figure 11. The wake circulation distribution predicted using the panel method is plotted on the surface representing the spatial trace of the wing's trailing edge, for each angle of attack $\alpha$ and twist scaling factors $\varepsilon=1.0,0.75,0.5$ from left to right. The wing is flying from left to right, starting with the downstroke. The wake circulation distribution is an indication of both the momentum transfer to the fluid from the flapping wing and the wake vortex structure.

desired at an early design stage, it may be sufficient to consider the adverse pressure gradient as an indicator of possible flow separation and concern.

\subsection{Effect of Separation on Force Prediction}

Overall the forces predicted by the potential flow model are comparable and trend accurate to the Navier-Stokes method. The detailed differences in the time evolution of the vertical and horizontal forces illustrate several cases where the potential flow approximation lacks the requisite physics to fully model the flow. The less aggressive cases, with at most moderate separation, are adequately modeled using potential flow theory, while the trends are captured approximately in the more aggressive separation cases. For all of the cases with a moderate or high wing twist parameter $(\epsilon=0.5,0.75)$, the potential flow consistently over-predicts the maximum vertical force. This is likely due to the lack of viscous modeling in the potential flow which prevents the method from representing the effective thickening of the airfoil as well as the modeling of flow separation. This close agreement between the potential flow model and the Navier-Stokes model, even in the presence of moderate separation occurs as a result of the nature of the flow separation. Two possibilities exist for explaining the unexpectedly good agreement in cases with separation. The first, is that much of the flow separation may be characterized by a subsequent re-attachment at downstream locations. This flow re-attachment may be promoted by the oscillatory nature of the wing flapping motions that promote the 
growth of a suction side vortex structure during the initial downstroke portion of the flapping cycle, with a shedding of this structure towards the end of the downstroke. Although not modeled in the potential flow solver, the effect of this attached suction side vortex may be modeled implicitly through the satisfaction of the Kutta condition at the trailing edge. In essence, the wing bound circulation, although inadequately modeled, may actually have the correct magnitude due to the satisfaction of the trailing edge condition. The second possibility for the unexpectedly good agreement between the panel method and the NavierStokes simulation when the flow is separated, is the contribution of the unsteady added mass effects in the force prediction. It is likely that these two effects act in unison in the cases we considered, but may act individually in other cases. Therefore, we expect good predictive capability from the panel method when there is flow separation if the flow re-attaches on the wing before the trailing edge and when the reduced frequency is high.

The force traces illustrate another feature of the viscous-inviscid modeling discrepancy. In the second half of the downstroke of more aggressive cases $(\epsilon=0.5,0.75)$, the potential flow method consistently over-predicts the vertical forces. In some cases the over-prediction is more pronounced than others. This can once again be attributed to the lack of viscous modeling in the potential flow model. There are two contributing factors to the viscous effects on the vertical force attenuation. First, the actual flow starts to separate from the wing at mid-downstroke. This flow separation causes a loss of vertical force due to the reduced circulation or flow turning. The flow separation is not captured by the potential flow approach and therefore, discrepancies will appear in these regimes. Secondly, the viscous boundary layers surrounding the wing cause an effective thickening of the airfoil at low Reynolds numbers. The boundary layers cause an effective displacement of the flow from the surface of the airfoil, reducing the ability of the airfoil to turn the flow and produce lift. This viscous boundary layer effect is not modeled in the potential flow.

The horizontal forces predicted by FastAero are also larger than those predicted by the Navier-Stokes simulation. The potential flow method consistently predicts larger thrust forces than the Navier-Stokes simulation results. Despite the trend accuracy, the lack of viscous modeling here is a critical deficit in the method's utility as a design tool, since accurate prediction of the drag and thrust is critical to the overall force balance and flight mechanics. At early stages of the design process, the accurate representation and prediction of the power required for flight derives directly the the capability to accurately predict flight forces. As a result, we recommend a viscous correction be considered for any potential flow method, to at minimum permit a reasonable horizontal force prediction.

\subsection{Wake structure}

For most cases, the wake structure predicted by the FastAero results is a double ladder configuration. This indicates that the downstroke is heavily loaded. The double ladder structure also implies that the outboard wing is loaded more than the inboard wing during the downstroke. These FastAero wakes are indicative of the general structure of the wake and may be compared to experimental results to assess the accuracy of the simulation and the efficacy of the design. The wake from the Navier-Stokes simulations indicates viscous effects may be critical in accurately predicting the actual wake. In these simulations, the wake has distinct tip structures, but the general behavior of the flow is much more complicated than that predicted by the potential flow. 


\subsection{Leading edge geometry}

The results of the simulations show the importance of wing leading edge geometry and the potential for designing effective leading edges for flapping vehicles using either method. The panel method predicts a significant leading edge pressure jump and subsequent adverse pressure gradient on the test cases - a strong indication of pending flow separation. The Navier-Stokes simulations confirm the suspicion that the flow separates from the leading edge. While this separation leads to a bound vortex which subsequently sheds into the wake, the leading edge shape can be prescribed to reduce the magnitude of or completely eliminate the pressure jump. This alignment or reshaping of the leading edge of the flapping wing may allow for vehicles with substantially lower leading edge separation. In related studies, we observe that the FastAero pressure differential predictions for geometrically accurate bat wings in flight [18] do not have a significant leading edge pressure jump. This is a direct result of the shape of the bat wing's leading edge. In bat flight, the wing-muscle-skeletal system is able to present an aligned or nearly aligned wing leading edge to the flow, thereby reducing the suction peak and likely resulting in a lower susceptibility to leading edge separation. While leading edge angle modulations are not guaranteed to eliminate flow separation, wing leading edge shape is recommended for further study.

\section{CONCLUSIONS}

We have numerically simulated a set of flapping wing problems using both a low-order potential flow model and a high-order accurate Navier-Stokes solver. The simulations confirm the challenges in analyzing and designing effective flapping wings. While our cost effective potential flow method produces accurate results for attached flow cases, it remains only trend relevant once separated flow dominates. While trend relevance is adequate for preliminary design, it may reduce the quality of the wing design if left unchecked. It is therefore critical to accompany any inviscid design predictions with a viscous simulation to confirm the predicted behavior. In essence, this study has confirmed our multi-fidelity approach while also demonstrating the importance of the high fidelity simulations.

\section{REFERENCES}

1. Larijani R, DeLaurier J. A nonlinear aeroelastic model for the study of flapping wing flight. Fixed and Flapping Wing Aerodynamics for Micro Air Vehicle Applications edited by E. T. J. Mueller, AIAA: Reston, VA, 2001; 399-428.

2. Katz J, Plotkin A. Low Speed Aerodynamics. Cambridge University Press: Cambridge, 2001.

3. Jones K, Lund T, Platzer M. Experimental and computational investigation of flapping wing propulsion. Fixed and Flapping Wing Aerodynamics for Micro Air Vehicle Applications, edited by E. T. J. Mueller, AIAA, Reston, VA, 2001; 307-336.

4. Wang J. Dissecting insect flight. Annual Review of Fluid Mechanics 2005; 37:183-210.

5. Shyy W, Liu H. Flapping wings and aerodynamic lift: The role of leading-edge vortices. AIAA Journal 2007; 45:2817-2819.

6. Willis D, Israeli E, Persson PO, Drela M, Peraire J, Swartz S, Breuer K. A computational framework for fluid structure interaction in biologically inspired flapping flight. 18th AIAA Computational Fluid Dynamics Conference, Miami, FL, 2007. AIAA-2007-3803.

7. Pennycuick C. Bird flight performance: a practical calculation manual. Oxford University Press: New York, New York, 1989. 
8. Rayner J. A vortex theory of animal flight. part 1. the vortex wake of a hovering animal. Journal of Fluid Mechanics 1979; 91:697-730.

9. Hall K, Pigott S, Hall S. Power requirements for large-amplitude flapping flight. Journal of Aircraft May-June 1998; 35(3):352-361. AIAA 35th Aerospace Science Meeting / Nonlinear Dynamical Systems Symposium, RENO, NEVADA, JAN 06-10, 1997.

10. DeLaurier J. An aerodynamic model for flapping-wing flight. Aeronautical Journal of the Royal Society April 1993; 97:125-130.

11. Ahmadi A, Widnall S. Energetics and optimum motion of oscillating lifting surfaces of finite span. Journal of Fluid Mechanics 1986; 162:261-282.

12. Albano E, Rodden W. A doublet-lattice method for calculating lift distributions on oscillating surfaces in subsonic flows. AIAA Journal 1969; 7(2):279-285.

13. Lan C. The unsteady quasi-vortex-lattice method with applications to animal propulsion. Journal of Fluid Mechanics 1979; 93:747-765.

14. Vest M, Katz J. Aerodynamics of a flapping-wing micro uav. 37th Aerospace Sciences Meeting in Reno, $N V, 1999$. AIAA 99-0994.

15. Smith M, Wilkin $\mathrm{P}$, Williams $\mathrm{M}$. The advantages of an unsteady panel method in modelling the aerodynamic forces on rigid flapping wings. $J$ Exp Biol 1996; 199(5):1073-1083. URL http://jeb.biologists.org/cgi/content/abstract/199/5/1073.

16. Willis D, Israeli E, Peraire J. Computational investigation and design of compliant membrane wings for biologically inspired flight vehicles. Proceedings of ICAS 2008, Anchorage, Alaska, 2008.

17. Tuncer I, Platzer M. Thrust generation due to airfoil flapping. AIAA Journal February 1996; 34(2):324331.

18. Willis DJ, Israeli ER, Persson PO, Drela M, Peraire J. Multifidelity approaches for the computational analysis and design of effective flapping wing vehicles. 46th AIAA Aerospace Sciences Meeting and Exhibit, Reno, Nevada, 2008. AIAA-2008-518.

19. Fritz T, Long N. Object-oriented unsteady vortex lattice method for flapping flight. Journal of Aircraft $2004 ;$ 41(6):1275-1290.

20. Neef M, Hummel D. Euler solutions for a finite-span flapping wing. Fixed and Flapping Wing Aerodynamics for Micro Air Vehicle Applicatons, edited by E. T. J. Mueller, AIAA, Reston, VA, 2001; 429-451.

21. Stanford B, Beran P. Analytical sensitivity analysis of an unsteady vortex-lattice method for flapping-wing optimization. Journal of Aircraft 2010; 47(2).

22. Palacios R, Murua J, Cook R. Structural and aerodynamic models in nonlinear flight dynamics of very flexible aircraft. AIAA Journal 2010; 48(11).

23. Willis DJ, Peraire J, White JK. A combined pFFT-multipole tree code, unsteady panel method with vortex particle wakes. Internat. J. Numer. Methods Fluids 2007; 53(8):1399-1422.

24. Hess JL. The problem of three-dimensional lifting flow and its solution by means of surface singularity distribution. Comput. Methods Appl. Mech. Engrg. 1974; 4:183-319.

25. Morino L, Kuo CC. Subsonic potential aerodynamics for complex configurations: A general theory. AIAA Journal 1974; 12(2):191-197.

26. Newman JN. Distribution of sources and normal dipoles over a quadrilateral panel. J. Engrg. Math. 1985; 20:113-126.

27. Strang G, Fix G. An Analysis of the Finite Element Method. Prentice Hall: London, 1973.

28. Leonard A. Computing three-dimensional incompressible flows with vortex elements. Ann. Rev. Fluid Mech. 1985; 17:523-559.

29. Gharakhani A, Ghoniem AF. Three-dimensional vortex simulation of time dependent incompressible internal viscous flows. J. Comput. Phys. 1997; 134(1):75-95.

30. Rehbach C. Calcul numérique d'écoulements tridimenssionnels instationnaires avec nappe tourbillonnaire. La Recherche Aérospatiale 1977; 5:289-298.

31. SG Voutsinas KR MA Belessis. Investigation of the yawed operation of wind turbines by means of a vortex particle method. AGARD-CP-552 FDP Symposium on Aerodynamics and Aeroacoustics of rotorcraft, Berlin, Germany, 1995. Paper 11.

32. Phillips J, White J. A precorrected-fft method for electrostatic analysis of complicated 3-d structures. IEEE Transactions On Computer Aided Design of Integrated Circuits and Systems 1997; 16.

33. Saad Y, Schultz MH. GMRES: a generalized minimal residual algorithm for solving nonsymmetric linear systems. SIAM J. Sci. Statist. Comput. 1986; 7(3):856-869.

34. Greengard L. The Rapid Evaluation of Potential Fields in Particle Systems. MIT Press: Cambridge, 1988.

35. Appel AW. An efficient program for many-body simulation. SIAM J. Sci. Stat. Comput. 1985; 6:85-103.

36. Crighton D. The kutta condition in unsteady flow. Annual Review of Fluid Mechanics January 1985; 17:411-445.

37. Peraire J, Persson PO. The compact discontinuous Galerkin (CDG) method for elliptic problems. SIAM J. Sci. Comput. 2008; 30(4):1806-1824. 
38. Persson PO, Peraire J. An efficient low memory implicit DG algorithm for time dependent problems. 44th AIAA Aerospace Sciences Meeting and Exhibit, Reno, Nevada, 2006. AIAA-2006-0113.

39. Persson PO, Peraire J. Newton-GMRES preconditioning for discontinuous Galerkin discretizations of the Navier-Stokes equations. SIAM J. Sci. Comput. 2008; 30(6):2709-2733.

40. Persson PO. Scalable parallel Newton-Krylov solvers for discontinuous Galerkin discretizations. 47th AIAA Aerospace Sciences Meeting and Exhibit, Orlando, Florida, 2009. AIAA-2009-606.

41. Persson PO, Peraire J, Bonet J. A high order discontinuous Galerkin method for fluid-structure interaction. 18th AIAA Computational Fluid Dynamics Conference, Miami, Florida, 2007. AIAA-2007-4327.

42. Nguyen NC, Persson PO, Peraire J. RANS solutions using high order discontinuous Galerkin methods. 45th AIAA Aerospace Sciences Meeting and Exhibit, Reno, Nevada, 2007. AIAA-2007-914.

43. Persson PO, Bonet J, Peraire J. Discontinuous Galerkin solution of the Navier-Stokes equations on deformable domains. Comput. Methods Appl. Mech. Engrg. 2009; 198(17-20):1585-1595.

44. Uranga A, Persson PO, Drela M, Peraire J. Implicit large eddy simulation of transition to turbulence at low reynolds numbers using a discontinuous galerkin method. International Journal for Numerical Methods in Engineering 2011; 87(1-5):232-261.

45. Persson PO, Strang G. A simple mesh generator in Matlab. SIAM Rev. 2004; 46(2):329-345 (electronic).

46. Morgan K, Peraire J. Unstructured grid finite element methods for fluid mechanics. Inst. of Physics Reviews 1998; 61(6):569-638.

47. Persson PO, Peraire J. Curved mesh generation and mesh refinement using Lagrangian solid mechanics. 47th AIAA Aerospace Sciences Meeting and Exhibit, Orlando, Florida, 2009. AIAA-2009-949.

48. Cockburn B, Shu CW. Runge-Kutta discontinuous Galerkin methods for convection-dominated problems. J. Sci. Comput. 2001; 16(3):173-261.

49. Roe PL. Approximate Riemann solvers, parameter vectors, and difference schemes. J. Comput. Phys. 1981; 43(2):357-372.

50. Alexander R. Diagonally implicit Runge-Kutta methods for stiff o.d.e.'s. SIAM J. Numer. Anal. 1977; 14(6):1006-1021.

51. Karypis G, Kumar V. METIS serial graph partitioning and fill-reducing matrix ordering Http://glaros.dtc.umn.edu/gkhome/metis/metis/overview. 